\title{
A General Outlook at the Pathogenesis of COPD
}

\author{
Maria Benito* \\ Medical Research, Trinity College Dublin, Ireland \\ *Corresponding author: Maria Benito, Medical Research, Trinity College Dublin, Ireland. \\ To Cite This Article: Maria Benito. A General Outlook at the Pathogenesis of COPD . Am J Biomed Sci \& Res. 2019 - 3(5). AJBSR.MS.ID.000708. \\ DOI: 10.34297/AJBSR.2019.03.000708
}

Received: June 25, 2019 | Published: July 03, 2019

\begin{abstract}
Chronic obstructive pulmonary disease (COPD) is characterized by deregulated chronic inflammation of the airways and persistent airflow obstruction, leading to emphysematous destruction of lung tissue and declining pulmonary function. There is a raise in infiltrated inflammatory cells in the lungs of COPD patients. Many processes are involved including damaged repair process that accelerates the development of the disease. Inflammatory mediators and dysfunctional airway smooth muscle (ASM) also play a role in COPD. Proteases and growth factors triggered by infectious pathogens, air pollution or second-hand smoke involved in the development of local and systemic immune response are upregulated during exacerbations. The role of innate immunity in COPD pathogenesis has been implicated in acute exacerbation that together with elevated interleukin (IL) 1-like cytokines suggests that inflammasomes play a role in the pathogenesis of COPD.
\end{abstract}

Keywords: Chronic Obstructive Pulmonary Disease; Inflammation; Inflammatory Infiltrated Cells; Exacerbation; Cytokines; Biomarkers; Senescence; Autophagy; Inflammasomes; Autoimmunity

Abbreviations: ASM: Airway Smooth Muscle; COPD: Chronic Obstructive Pulmonary Disease; CRP: C-Reactive Protein; IL: Interleukin; NLRs: Nucleotide-Binding Domain Leucine-Rich Repeat-Containing; Receptors; PRRs : Pattern Recognition Receptors; RLRs : RIG-I-Like Receptors; ROS : Reactive Oxygen Species; TLRs : Toll-Like Receptors

\section{Introduction}

Chronic obstructive pulmonary disease (COPD) is a pathological disorder characterized by deregulated chronic inflammation of the airways and persistent airflow obstruction [1,2], which lead to emphysematous destruction of lung tissue and deterioration of the pulmonary function [3]. The characteristics of COPD include infiltration of neutrophils, macrophages, B and T lymphocytes, and dendritic cells that release inflammatory cytokines, proteases, and growth factors responsible for the structural changes in the lung. It also comprises mucociliary dysfunction, apoptosis, and structural changes in the airways causing emphysema, and extrapulmonary systemic effects [4], with a calculated prevalence of $10 \%$ in over forty years old adults. Clinical features of COPD include persistent pulmonary inflammation, obstructive bronchiolitis, chronic bronchitis, emphysema and loss of alveolar tissue. Conventional therapies work only as palliative treatments. Respiratory infections increase of cardiovascular risk, lung cancer, pulmonary hypertension, and depression are some of the most common complications caused by this disorder. COPD is a significant leading cause of deaths worldwide, and by 2020 , it is expected to be the third leading cause of deaths worldwide [5]. Cigarette smoking is the major etiologic and risk factor for COPD [6]. Aging is considered a critical factor in the development of COPD [7]. It's been shown that the incidence of COPD increases with aging, with a peak observed in patients aged 65-74 years [8,9].

The pathogenesis of COPD remains largely unclear. It is known that emphysema is characterized by the presence of humoral and cellular autoimmune responses against elastin [10], and bronchial smooth muscle cell hyperplasia [11], showing evidence for a role of autoimmunity in COPD pathogenesis, with a prominent role of inflammation. Dysregulated inflammation, dysfunction in airway smooth muscle (ASM), imbalance in the proteolysis/ antiproteolysis equilibrium and in the repair process, and different epigenetic mechanisms -including DNA methylation, decreased levels of histone deacetylases and reduced microRNAs levelsare mechanisms that contribute to COPD pathophysiology [12]. Inflammatory cells influence cell destruction, hyperplasia of smooth muscle cells, and subepithelial fibrosis seen in COPD. It has been hypothesized that inflammatory cells infiltrate the bronchial mucosa and lung parenchyma in COPD lungs, affecting the airway destruction and remodeling by secreting enzymes and inflammatory cytokines or by indirect interference regulating other cellular functions [13], promoting tissue damage and reconstruction. However, the mechanism of participation of different inflammatory mediators is not completely known. Currently, no good biomarkers 
of remodeling are available, and imaging techniques are not sensitive enough to directly visualize the remodeling changes in the airways.

Cytokines have a controversial and sometimes opposite role in COPD; the elimination of a single one may further deregulate the inflammatory process. Cells and mediators of immunity, and reactive oxygen species (ROS) also contribute to inflammation [14]. Innate and adaptive immune systems are involved in the development of chronic inflammation leading to COPD. The role of innate immunity in COPD pathogenesis has been implicated in the induction and acute exacerbation $[15,16]$, with Interleukin (IL) 1-like cytokines increased in COPD patients suggesting the role of inflammasomes -intracellular multiproteines complexes that activates proinflammatory caspases- in the pathogenesis of COPD that lead to the production of IL-1 $\beta$ and IL-18 in response to pathogenesis $[15,16]$. Innate immune cells recognize microbial pathogens or damage-associated molecular patterns or recognition of innate immune cells, which activate the inflammasome through several families of pattern recognition receptors (PRRs) expressed in innate immune cells, including Toll-like receptors (TLRs), nucleotide-binding domain leucine-rich repeat-containing receptors (NLRs), C-type lectin receptors, and RIG-I-like receptors $[15,16]$. TLRs are expressed on alveolar macrophages, lymphocytes, dendritic cells, and bronchial epithelial cells $[15,16]$.

COPD exacerbations are defined as sustained worsening of a patient's condition associated with respiratory -dyspnea and productive cough- and non-respiratory -fatigue and malaisesymptoms [17] beyond normal day-to-day variations that is acute in onset. Several biomarkers of inflammation can help to identify exacerbations most likely to respond to oral corticosteroids and antibiotics, and patients with a frequent exacerbation phenotype, which requires a preventative treatment [18]. While mild exacerbations can be managed with inhaled bronchodilators, for worsening symptoms, while moderate exacerbations need treatment with antibiotics and/or corticosteroids, and severe exacerbations require hospitalization [17]. The heterogeneity in COPD clinical manifestations, outcomes, and responses to treatment [19] is useful to classify COPD into specific phenotypes. Many exacerbations of COPD involve bacterial or viral respiratory infections [20]. Accelerated cell senescence and insufficient autophagy -significantly deregulated in the cells from COPD patients- increase the accumulation of damaged cells [21]. Cellular senescence has been widely implicated in the pathogenesis of COPD, presumably by impairing cell repopulation and by aberrant cytokine secretion in the senescence-associated secretory phenotype. Autophagy is a process of lysosomal self-degradation that maintains a homeostatic balance between the synthesis, degradation, and recycling of cellular proteins.

Peripheral blood eosinophil count was shown to be a valid biomarker for sputum eosinophil-associated exacerbations [22]. Patients with COPD and evidence of eosinophilic airway inflammation respond well to corticosteroid therapy [23]. C-reactive protein (CRP) is another potentially useful biomarker for predicting which exacerbations may benefit from antibiotic therapy and for selecting those that might resolve without antibiotic intervention [24]. Procalcitonin may not be sufficiently sensitive for use as a biomarker for response to antibiotics in patients with COPD exacerbations [24]. Therefore, the use of CRP as a biomarker could help to avoid unnecessary use of antibiotic therapy that can lead to adverse effects and development of bacterial resistance [25]. In addition to bronchodilators, macrolide azithromycin, taken daily for 1 year in addition to usual therapy, demonstrated a significant reduction in the risk of exacerbations in patients with COPD at increased risk of exacerbation [26]. Furthermore, there is evidence that pneumococcal and annual influenza vaccinations reduce the risk of exacerbation and hospitalization in patients with COPD [2729]. Other mechanisms further upregulated during exacerbations -triggered by infectious pathogens, air pollution or second-hand smoke- include amplification of inflammatory process.

\section{Conclusion}

Inflammation plays a key role in the development of COPD. Other mechanisms such as senescence, autophagy or repair process are dysregulated in COPD. Despite all the current knowledge, there is still a long way to go to further unveil the pathogenesis of COPD, and find useful biomarkers for diagnosis, response to treatments and outcome.

\section{References}

1. Polosukhin VV, Richmond BW, Du RH, Cates JM, Wu P, et al. (2017) Secretory IgA deficiency in individual small airways is associated with persistent inflammation and remodeling. Am J Respir Crit Care Med 195(8): 1010-1021.

2. Richmond BW, Du RH, Han W, Benjamin JT, van der Meer R, et al. (2018) Bacterial-derived neutrophilic inflammation drives lung remodeling in a mouse model of COPD. Am J Respir Cell Mol Biol 58(6): 736-744.

3. Vestbo J, Hurd SS, Agustí AG, Jones PW, Vogelmeier C, et al. (2013) Global strategy for the diagnosis, management, and prevention of chronic obstructive pulmonary disease: GOLD executive summary. Am J Respir Crit Care Med 187(4): 347-365.

4. Hogg JC, Timens W (2009) The pathology of chronic obstructive pulmonary disease. Annu Rev Pathol 4: 435-459.

5. Weiner A, Chen HV, Liu CL, Rahat A, Klien A, et al. (2012) Systematic dissection of roles for chromatin regulators in a yeast stress response. PLoS Biol 10: e1001369.

6. Caramori G, Casolari P, Barczyk A, Durham AL, Di SA, et al. (2016) COPD immunopathology. Semin Immunopathol 38(4): 497-515.

7. Ito K, Barnes PJ (2009) COPD as a disease of accelerated lung aging. Chest 135: $173-180$

8. Mannino DM, Homa DM, Akinbami LJ, Ford ES, Redd SC (2002) Chronic obstructive pulmonary disease surveillance-United States, 19712000. MMWR Surveill Summ 51: 1-16.

9. Faner R, Rojas M, Macnee W, Agustí A (2012) Abnormal lung aging in chronic obstructive pulmonary disease and idiopathic pulmonary fibrosis. Am J Respir Crit Care Med 186: 306-313.

10. Bhat TA, Panzica L, Kalathil SG, Thanavala Y (2015) Immune dysfunction in patients with chronic obstructive pulmonary disease. Ann Am Thorac Soc 12(Suppl 2): S169-S175.

11. Hirota N, Martin JG (2013) Mechanisms of airway remodeling. Chest 144(3): 1026-1032.

12. Zong DD, Ouyang RY, Chen P (2015) Epigenetic mechanisms in chronic 
obstructive pulmonary disease. Eur Rev Med Pharmacol Sci 19(5): 844 856.

13. Wang Y, Xu J, Meng Y, Adcock IM, Yao X (2018) Role of inflammatory cells in airway remodeling in COPD. Int J Chron Obstruct Pulmon Dis 13: 3341-3348.

14. Kirkham PA, Barnes PJ (2013) Oxidative stress in COPD. Chest 144(1): 266-273.

15. Rycroft CE, Heyes A, Lanza L, Becker K (2012) Epidemiology of chronic obstructive pulmonary disease: a literature review. Int J Chron Obstruct Pulmon Dis 7: 457-494.

16. Rovina N, Koutsoukou A, Koulouris NG (2013) Inflammation and immune response in COPD: where do we stand? Mediators Inflamm 413735.

17. Rodriguez-Roisin R (2000) Toward a consensus definition for COPD exacerbations. Chest 117(5 Suppl 2): 398S-401S.

18. Pavord ID, Jones PW, Burgel PR, Rabe KF (2016) Exacerbations of COPD. Int J Chron Obstruct Pulmon Dis 11 Spec Iss: 21-30.

19. Han MK, Agusti A, Calverley PM, Celli BR, Criner G, et al. (2010) Chronic obstructive pulmonary disease phenotypes: the future of COPD. Am J Respir Crit Care Med 182(5): 598-604.

20. Sethi S, Murphy TF (2008) Infection in the pathogenesis and course of chronic obstructive pulmonary disease. N Engl J Med 359(22): 23552365 .

21. Kuwano K, Araya J, Hara H, MinagawaS, Takasaka N, et al. (2016) Cellular senescence and autophagy in the pathogenesis of chronic obstructive pulmonary disease (COPD) and idiopathic pulmonary fibrosis (IPF). Respir Investig 54(6): 397-406.
22. Bafadhel M, McKenna S, Terry S, Mistry V, Reid C, et al. (2011) Acute exacerbations of chronic obstructive pulmonary disease: identification of biologic clusters and their biomarkers. Am J Respir Crit Care Med 184(6): 662-671.

23. Brightling CE, Monteiro W, Ward R, Parker D, Morgan MD, et al. (2000) Sputum eosinophilia and short-term response to prednisolone in chronic obstructive pulmonary disease: a randomised controlled trial. Lancet 356(9240): 1480-1485.

24. Daniels JM, Schoorl M, Snijders D, Knol DL, Lutter R, et al. (2010) Procalcitonin vs C-reactive protein as predictive markers of response to antibiotic therapy in acute exacerbations of COPD. Chest 138(5): 11081115 .

25. Herath SC, Poole P (2013) Prophylactic antibiotic therapy for chronic obstructive pulmonary disease (COPD) Cochrane Database Syst Rev 11: CD009764.

26. (2015) Global Initiative for Chronic Obstructive Lung Disease [homepage on the Internet] Global strategy for the diagnosis, management, and prevention of chronic obstructive pulmonary disease.

27. Albert RK, Connett J, Bailey WC (2011) Azithromycin for prevention of exacerbations of COPD. N Engl J Med 365(8): 689-698.

28. Poole PJ, Chacko E, Wood-Baker RW, Cates CJ (2000) Influenza vaccine for patients with chronic obstructive pulmonary disease. Cochrane Database Syst Rev 4: CD002733.

29. Walters JA, Smith S, Poole P, Granger RH, Wood-Baker R (2010) Injectable vaccines for preventing pneumococcal infection in patients with chronic obstructive pulmonary disease. Cochrane Database Syst Rev 11: CD001390. 